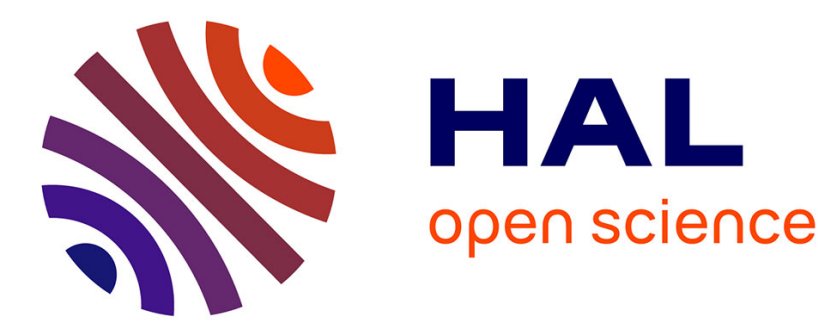

\title{
La vulnérabilité dans la jurisprudence de la Cour européenne et de la Cour interaméricaine des droits de l'homme
}

\author{
Marie Rota
}

\section{- To cite this version:}

Marie Rota. La vulnérabilité dans la jurisprudence de la Cour européenne et de la Cour interaméricaine des droits de l'homme. Cahiers de la recherche sur les droits fondamentaux , 2020, 10.4000/crdf.6422 . hal-03007474

\section{HAL Id: hal-03007474 \\ https://hal.science/hal-03007474}

Submitted on 24 Nov 2020

HAL is a multi-disciplinary open access archive for the deposit and dissemination of scientific research documents, whether they are published or not. The documents may come from teaching and research institutions in France or abroad, or from public or private research centers.
L'archive ouverte pluridisciplinaire HAL, est destinée au dépôt et à la diffusion de documents scientifiques de niveau recherche, publiés ou non, émanant des établissements d'enseignement et de recherche français ou étrangers, des laboratoires publics ou privés. 


\title{
La vulnérabilité dans la jurisprudence de la Cour européenne et de la Cour interaméricaine des droits de l'homme
}

\author{
Marie ROTA \\ Maître de conférences en droit public à l'université de Lorraine \\ Institut de recherches sur l'évolution de la nation et de l'État (IRENEE, EA 7303)
}

I. Le recours à la vulnérabilité: une démarche relativement similaire

A. Un recours croissant dénué de définition préalable

$B$. La résilience: une finalité commune

II. La vulnérabilité: un concept reflétant une conception différente des droits humains adoptée par les Cours
A. La lecture libérale de la vulnérabilité par la Cour européenne
B. La lecture sociale de la vulnérabilité par la Cour interaméricaine

Dans les récentes manifestations scientifiques consacrées à la vulnérabilité, qu'il s'agisse des deux colloques de 2018 organisés par les juristes Cathy Pomart et François

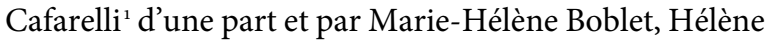
Marche et Nadine Proia-Lelouey ${ }^{2}$, chercheuses en littérature, sociologie et psychologie respectivement d'autre part, ou encore de la journée d'étude organisée par Mathias Couturier, Delphine Bazin-Beust, Samuel Etoa, Aurore Catherine et Laurence Fin-Langer en $202 \mathrm{O}^{3}$, les contributeurs n'ont eu de cesse de souligner l'accroissement du recours au concept en droit depuis une vingtaine d'années, sans qu'on puisse néanmoins en saisir réellement les contours. De fait, le constat dressé par Laurence Burgorgue-Larsen en 2014 semble toujours être d'actualité: «[s]i le concept a fleuri, sa conceptualisation quant à elle n'a pas suivi» ${ }^{4}$. Facteur de flou, son incertitude a été largement soulignée 5 . Diane Roman, dans le rapport de synthèse de cette première manifestation, ajoute une difficulté supplémentaire: le fait que ce concept «se superpose souvent à d'autres catégories juridiques plus

1. Ce colloque, intitulé "Vulnérabilité et droits fondamentaux», s'est tenu les 19 et 20 avril 2018 à l'université de la Réunion et a donné lieu à la publication d'un dossier dans la Revue des droits et libertés fondamentaux, 2019, revue en ligne: http://www.revuedlf.com/dossier/colloquevulnerabilite-et-droits-fondamentaux-19-20-avril-2018-universite-de-la-reunion.

2. Ce colloque interdisciplinaire, intitulé «Penser / exposer la vulnérabilité» auquel nous avons participé, s'est tenu du 29 novembre au ${ }^{\text {er }}$ décembre 2018 à l'université de Caen Normandie (voir https://vulnerabilite.sciencesconf.org/resource/page/id/11).

3. Cette journée d'étude, intitulée «Vulnérabilités et droit, regards croisés», s'est tenue le 7 février 2020 à l'université de Caen Normandie (voir http://www.unicaen.fr/recherche/mrsh/vss/6438).

4. L. Burgorgue-Larsen, «La vulnérabilité saisie par la philosophie, la sociologie et le droit. De la nécessité d'un dialogue inter-disciplinaire», in La vulnérabilité saisie par les juges en Europe, L. Burgorgue-Larsen (dir.), Paris, Pedone, 2014, p. 241.

5. Nous pouvons ici rappeler les propos de Florence Faberon: «Vulnérabillité, vulnérables: ces notions avec leur caractère de "mots-éponge" ont un aspect emblématique, un pouvoir de rassemblement, un caractère évocateur en raison de leur malléabilité intrinsèque. Attirantes, elles n'en sont pas moins limitées. Elles sont plus subjectives qu'objectives. Elles ont surtout une faiblesse évidente: elles sont sans contour défini» (F. Faberon, «Vulnérabilité et besoin dans le droit de l'aide et de l'action sociales», in Effectivité des droits et vulnérabilité de la personne, E. Paillet, P. Richard (dir.), Bruxelles, Bruylant, 2014, p. 52). 
anciennes ${ }^{6}$, telles que celles « de pauvreté et d'exclusion sociale», de "dépendance, inaptitude, invalidité et handicap» ou encore d' «incapacité» ${ }^{7}$.

S'agissant de la définition du concept, elle fait aussi largement défaut, le législateur et les juges préférant "procéder par énumération de catégories de personnes vulnérables plutôt que par définition de la vulnérabilité ${ }^{8}$. La doctrine s'y est cependant essayée. Pour Xavier Lagarde, « sous l'angle du droit, la personne vulnérable est celle qui n'est pas en mesure d'exercer les attributs de la personnalité juridique ${ }^{9}$. Selon Diane Roman, «la vulnérabilité peut se définir comme l'état d'une personne qui, en raison d'un contexte donné, ne peut, en droit ou en fait, jouir de l'autonomie suffisante pour exercer pleinement ses droits fondamentaux $»^{10}$. Nous souscrivons, quant à nous, à cette dernière définition dans la mesure où elle insiste sur le contexte qui entoure cet état de fait - la vulnérabilité auquel le droit vient attacher des conséquences juridiques, et donc sur son caractère réversible.

Le second trait commun de ces études consacrées au recours à la vulnérabilité en droit est de souligner les risques qui l'accompagnent. Comme le résume bien Caroline Boiteux-Picheral, trois écueils peuvent apparaître:

Le premier [...] est celui de la différenciation, qui heurterait l'universalité des droits de l'homme et l'égalité des sujets de droit. Le deuxième est celui de la catégorisation avec d'une part, le risque de verser dans un essentialisme qui [...] réduirait les personnes concernées à un seul attribut [...] et d'autre part, le risque d'entretenir alors des stéréotypes, sinon un processus de stigmatisation. Le troisième problème est celui du paternalisme, potentiellement attentatoire aux libertés des intéressés et de nature, le cas échéant, à les déresponsabiliser ${ }^{11}$.

C'est pourquoi la vulnérabilité est «une ressource à manier avec prudence $»^{12}$. Il semble alors opportun de s'interroger sur le fait de savoir si cette prudence est de mise s'agissant de la jurisprudence des deux Cours régionales de protection des droits humains que sont les Cours européenne et interaméricaine des droits de l'homme. Toutes deux n'hésitent pas, en effet, à recourir à la vulnérabilité, suivant une démarche qu'on pourrait qualifier de similaire (I). En conclure qu'il s'agirait là d'un concept identique serait cependant un jugement trop hâtif. Dans la mesure où la vulnérabilité s'inscrit dans le prolongement de la dignité humaine, elle-même appréhendée de manière fort différente d'un juge à l'autre, on peut affirmer qu'elle reflète une conception des droits humains qui, elle aussi, diffère (II).

\section{Le recours à la vulnérabilité : une démarche relativement similaire}

Les deux Cours régionales se saisissent du concept de vulnérabilité de manière croissante. Elles refusent cependant de s'enfermer dans une définition précise (A) tout en attachant à cette qualification juridique des faits des conséquences ayant la même finalité (B).

\section{A. Un recours croissant dénué de définition préalable}

Ni la Convention de sauvegarde des droits de l'homme et des libertés fondamentales, ni la Convention américaine relative aux droits de l'homme, ni leurs protocoles ne mentionnent le concept de vulnérabilité. Si la vulnérabilité est bien nommée et explicitement employée, ce n'est donc pas dès l'origine mais bien par les juges qui vont s'en saisir de manière progressive et à un rythme de plus en plus soutenu.

À ce titre, trois remarques s'imposent. Tout d'abord, les références au concept ne sont pas tout à fait concomitantes et surtout le nombre d'occurrences diffère. Pour ce qui est de la jurisprudence de la Cour européenne, la notion émerge en $1981^{13}$, mais la Cour ne s'y réfère alors que très rarement et de manière éparse. Ce n'est qu'à partir de 2001 qu'on observe une utilisation plus systématique ${ }^{14}$. Depuis, on retrouve des mentions régulières de la vulnérabilité dans la jurisprudence de la Cour, mais qui restent tout de même assez rares au regard du très grand nombre de décisions rendues par année. Une brève recherche sur la base de données Hudoc montre que sur les un peu moins de 124000 arrêts et décisions rendus jusqu'au 3 avril 2020, on ne trouve que 171 et 227 occurrences des termes

6. D. Roman, «Vulnérabilité et droits fondamentaux - Rapport de synthèse », Revue des droits et libertés fondamentaux, 2019, chron. 19, en ligne: http://www.revuedlf.com/droit-fondamentaux/dossier/vulnerabilite-et-droits-fondamentaux-rapport-de-synthese.

7. Ibid.

8. J.-Y. Carlier, «Des droits de l'homme vulnérable à la vulnérabilité des droits de l'homme, la fragilité des équilibres », Revue interdisciplinaire d'études juridiques, $\mathrm{n}^{\circ}$ 79, 2017, p. 184-185.

9. X. Lagarde, avant-propos au rapport annuel de la Cour de cassation, Les personnes vulnérables dans la jurisprudence de la Cour de cassation, Paris, La documentation française, 2009, p. 59, en ligne: https://www.courdecassation.fr/publications_26/rapport_annuel_36/rapport_2009_3408.

10. D. Roman, "Ils ne mouraient pas tous, mais tous étaient frappés". Le coronavirus, révélateur des ambiguïtés de l'appréhension juridique de la vulnérabilité», Revue des droits et libertés fondamentaux, 2020, chron. 15, en ligne: http://www.revuedlf.com/droit-administratif/ils-ne-mouraientpas-tous-mais-tous-etaient-frappes-le-coronavirus-revelateur-des-ambiguites-de-lapprehension-juridique-de-la-vulnerabilite.

11. C. Boiteux-Picheral, «Introduction - Vers une protection "systématisée” des personnes vulnérables? ", in La vulnérabilité en droit européen des droits de l'homme. Conception(s) et fonction(s), C. Boiteux-Picheral (dir.), Limal - Bruxelles, Anthemis - Nemesis, 2019 , p. 13.

12. M.-H. Soulet, «La vulnérabilité, une ressource à manier avec prudence», p. 7.

13. Cour EDH, 22 octobre 1981, Dudgeon c. Royaume-Uni, nº $7525 / 76$, spéc. $\$ 47,60$ et 62.

14. S. Besson, «La vulnérabilité et la structure des droits de l'homme. L’exemple de la jurisprudence de la Cour européenne des droits de l'homme», in La vulnérabilité saisie par les juges en Europe, p. 65. 
«vulnérabilité » et «vulnerability» ainsi que 717 occurrences du terme «vulnérable» ou «vulnerable» dans la section «en droit» de ces différents arrêts ou décisions ${ }^{15}$.

Pour ce qui est de la jurisprudence de la Cour interaméricaine, l'emploi du concept est plus tardif, puisqu'il n'est mentionné pour la première fois qu'en $1997^{16}$. Il faut cependant avoir à l'esprit que la Cour est plus jeune que sa consœur européenne puisqu'elle n'a vu le jour qu'en 1979 et a rendu sa première affaire au contentieux qu'en $1987^{17}$. Il s'agissait alors de l'une de ses premières décisions et plus précisément de la onzième rendue au fond de l'affaire (sur 401 décisions rendues jusqu'au 3 avril 2020). À titre de comparaison, la Cour européenne n'avait mentionné le concept qu'au bout de 300 décisions environ et surtout 21 années après la première. On ne retrouve donc pas de mention du concept dès l'origine de sa jurisprudence comme c'est le cas au niveau interaméricain. Il faut enfin signaler que la Cour interaméricaine fait une mention récurrente de la vulnérabilité dans ses différentes affaires ${ }^{18}$, alors qu'au niveau européen cela ne concerne que des cas particuliers $^{19}$.

Qu'il s'agisse de la Cour interaméricaine ou de la Cour européenne, ensuite, tout type de fragilité est envisagé. Il peut s'agir d'un état de vulnérabilité physique, psychologique, économique ou encore social. Sont qualifiés de «vulnérables» des enfants ${ }^{20}$, des personnes en fin de vie, des personnes malades, des femmes, des membres de minorités sexuelles ou encore de populations autochtones: celles et ceux que Rosmerlin Estupiñan-Silva appelle les personnes vulnérables en raison de leur «condition personnelle ${ }^{21}$. Les Cours peuvent aussi viser parfois des migrants ou déplacés forcés, des détenus et, pour ce qui est de la Cour interaméricaine, des dirigeants de partis politiques d'opposition, des défenseurs des droits humains, ou encore des journalistes, ce que l'auteure appelle les personnes vulnérables en raison "d'une situation spécifique $»^{22}$.

Il faut enfin préciser, et c'est l'objet de notre troisième remarque, qu'aucune des deux Cours ne donne de définition précise de la vulnérabilité. S'agissant de la Cour interaméricaine, elle en fait cependant une utilisation récurrente et détaillée, qui aboutit à ce qu'on puisse élaborer ce que Rosmerlin Estupiñan-Silva appelle un «test de vulnérabilité», qu'elle détaille en identifiant plusieurs éléments tels que «les causes sous-jacentes», «l'exposition aux pressions» ou encore «la sensibilité face à la menace ${ }^{23}$. À partir de là, elle en fait découler un «test de vulnérabilité ${ }^{24}$, tout en reconnaissant qu'il est encore «en construction ${ }^{25}$, la Cour ne s'enfermant pas dans une démarche précise.

C'est au même constat que se livre Céline Ruet en 2015, analysant le concept dans la jurisprudence de la Cour européenne des droits de l'homme. Selon elle, cette notion, «jamais définie, [...] s'accompagne encore de flou et d'ambiguïtés, malgré l'importance quantitative de ses occurrences et un ordonnancement qui reste partiel tout en étant en progression ${ }^{26}$. Quatre années plus tard, à l'occasion de l'examen de l'affaire Ilias et Ahmed c. Hongrie, cinq universitaires italiens agissant en tant que tiers intervenants ont explicitement demandé à la Cour de «fixer des principes pertinents » à l'égard de la vulnérabilité, en raison de l'absence de définition dont la notion pâtit et de son utilisation variable selon les contextes ${ }^{27}$. La Cour refuse cependant de s'y attarder, ne faisant que rappeler sa jurisprudence classique en matière de séjour de demandeurs d'asile en zone de transit ${ }^{28}$.

Ce commun refus peut être expliqué de différentes façons. La difficulté d'en saisir les contours en est une ${ }^{29}$. La «volonté [des Cours] de ne pas s'enfermer dans un schéma préétabli qui pourrait constituer une contrainte

15. Base de données Hudoc, https://hudoc.echr.coe.int, consultée le 3 avril 2020. Pour des données statistiques plus précises, se référer à S. Besson, "La vulnérabilité et la structure des droits de l'homme...», p. 65-66 et 81-85 ou encore C. Boiteux-Picheral, «Introduction...», p. 9-10.

16. Cour IDH, 17 septembre 1997, Loayza Tamayo c. Pérou, fond, série C, $\mathrm{n}^{\circ} 33, \$ 57$.

17. La Convention américaine est en effet entrée en vigueur le 18 juillet 1978, la première élection des juges a eu lieu le 22 mai 1979 et la nouvelle Cour a été convoquée le 29 juin 1979. Néanmoins, elle ne rend son premier avis qu'en 1982, et sa première affaire qu'en 1987 (Cour IDH, 26 juin 1987 , Velásquez Rodríguez c. Honduras, exceptions préliminaires, série $\mathrm{C}, \mathrm{n}^{\circ} 1$ ).

18. Voir, sur ce point, la très complète étude de R. Estupiñan-Silva, «La vulnérabilité saisie par la Cour interaméricaine», in La vulnérabilité saisie par les juges en Europe, p. 105.

19. Comme le constate Caroline Boiteux-Picheral, «rapportée au nombre total d'arrêts rendus par la Cour de Strasbourg chaque année, la proportion reste sans doute modeste» (C. Boiteux-Picheral, «Introduction...», p. 10).

20. La vulnérabilité de la personne mineure est la plus simple à caractériser dans la mesure où elle résulte d'une faiblesse de la personne «qui empêche a priori l'individu d'exercer convenablement l'ensemble des attributs de la personnalité juridique» (X. Lagarde, avant-propos au rapport annuel de la Cour de cassation, Les personnes vulnérables..., p. 64).

21. R. Estupiñan-Silva, «La vulnérabilité saisie par la Cour interaméricaine», p. 105.

22. Ibid., p. 106. Pour une autre analyse de la jurisprudence interaméricaine, voir, entre autres, S. García Ramírez, «La afectaciones a los derechos humanos de las personas vulnerables y las minorías. El papel de la Corte Interamericana de Derechos Humanos », in Diálogos constitucionales de Colombia con el mundo, J. C. Henao (dir.), Bogota, Universita del Externado, 2013, p. 495-502. Pour ce qui est de la Cour européenne, se référer à A. Palanco, «Les variations autour des formes de vulnérabilité reconnues en droit européen des droits de l'homme », in La vulnérabilité en droit européen des droits de l'homme..., p. 33-61.

23. R. Estupiñan-Silva, «La vulnérabilité saisie par la Cour interaméricaine», p. 94-104.

24. Ibid., p. 94-109.

25. Ibid., p. 90 et 108 .

26. C. Ruet, «La vulnérabilité dans la jurisprudence de la Cour européenne des droits de l'homme », Revue trimestrielle des droits de l'homme, 2015, p. 317.

27. Cour EDH, GC, 21 novembre 2019, Ilias et Ahmed c. Hongrie, $\mathrm{n}^{\circ}$ 47287/15, \$185.

28. Ibid., $\$ 191-192$.

29. Tous les auteurs qui se sont penchés sur le concept insistent sur sa polysémie. Voir, par exemple, S. Besson, «La vulnérabilité et la structure des droits de l'homme...", p. 60 
dans l'avenir $»^{30}$ en est une autre. Il faut par ailleurs rappeler que le concept de vulnérabilité est à la fois propre à la condition humaine (le vulnérable, selon le sens courant, est celui «qui peut être blessé $»^{31}$, soit toute personne) et consubstantiel aux droits humains ${ }^{32}$. Aussi, «le rôle pivot de la vulnérabilité dans la structure d'un droit de l'homme, ou du moins celui de la vulnérabilité générale ou standard, [...] fait que la Cour ne considère pas qu'elle doive l'expliquer ou justifier son utilisation en pratique ${ }^{33}$. Néanmoins, si cela peut apparaître acceptable s'agissant de l'utilisation de LA vulnérabilité, celle qui est consubstantielle à l'être humain, elle l'est beaucoup moins s'agissant de l'utilisation par la Cour DES vulnérabilités, celles qui sont situées, les vulnérabilités dites spécifiques ${ }^{34}$ ou encore particulières ${ }^{35}$. Une justification paraît en effet souhaitable du point de vue de la sécurité juridique, tant pour les États, pour qui la vulnérabilité est un facteur de contrainte ${ }^{36}$, que - et surtout - pour le justiciable, pour qui elle est un moyen d'obtenir un accès aux droits renforcé. Car, et c'est là le second point commun entre la jurisprudence des deux Cours qui nous permet de qualifier leur démarche de similaire, la finalité du recours à la vulnérabilité est commune.

\section{B. La résilience: une finalité commune}

S'il est un concept sur lequel les juristes insistent peu par rapport aux chercheurs des autres sciences humaines et sociales, c'est bien celui de résilience. Ce terme renvoie à la capacité d'une personne, voire d'une société, de surmonter une épreuve ${ }^{37}$, prolongeant en quelque sorte l'aphorisme de Nietzsche selon lequel «ce qui ne nous tue pas nous rend plus fort». Lors du colloque organisé en 2018 par des chercheuses en littérature, sociologie et psychologie, la plupart des intervenants y faisaient une référence constante, tout en soulignant le risque de tomber dans une certaine stigmatisation entre le «bon vulnérable» - le résilient - et le «mauvais vulnérable» - celui qui, au contraire, ne développe aucune velléité de résilience. La table ronde consacrée plus spécifiquement au droit, intitulée "Vulnérabilités nommées et innommées", y a fait figure de remarquable exception: à aucun moment le concept n'a été mentionné. La majorité des contributions des juristes relatives à la vulnérabilité en atteste également: une seule y fait, à notre connaissance, référence ${ }^{38}$.

Or il semble pourtant bien que le recours à la vulnérabilité en droit ait cette finalité. Elle permet, dans les deux cas, aux (catégories de) personnes ou aux groupes qualifiés de vulnérables ${ }^{39}$ de retrouver une certaine autonomie en vue de rendre leurs droits humains effectifs - et on retrouve ici le caractère nécessairement réversible de la vulnérabilité auquel nous faisions mention en introduction. Comme le constatent Mary Beloff et Laura Clérico :

L'argument de la vulnérabilité [...] met l'accent sur le fait que des personnes ou des groupes de personnes nécessitent l'intervention d'un tiers (l'État) pour les aider à sortir de l'état de vulnérabilité, car lorsqu'elles sont en situation d'exclusion, de marginalité, de subordination, elles ne disposent pas des mêmes outils [...] pour influencer et inverser la situation ${ }^{40}$.

Cette question, qui ressort peut-être davantage de la philosophie du droit, mérite cependant d'être posée pour comprendre la démarche des deux Cours. Car le droit, et plus précisément la jurisprudence des deux Cours qui impose ou incite $^{41}$ une adaptation des droits internes, a pour but de parvenir à une certaine résilience du vulnérable, via plusieurs procédés.

La facilitation de l'accès aux deux Cours est le premier. Les juges se livrent en effet à une lecture des conditions de recevabilité relativement souple à l'égard des personnes qualifiées de vulnérables ou à leurs représentants. C'est ce qui correspond en partie à ce que Samantha Besson

30. A. Palanco, «Les variations autour des formes de vulnérabilité... », p. 33.

31. Selon le dictionnaire de l'Académie française, $8^{\mathrm{e}}$ éd., en ligne: https://www.dictionnaire-academie.fr.

32. Selon Samantha Besson, pour qu'il y ait un droit de l'homme, trois éléments doivent être caractérisés: "(i) des intérêts objectifs fondamentaux, (ii) menacés de manière à rendre leur protection nécessaire, (iii) dont la protection par des droits et obligations impose un fardeau non seulement faisable mais aussi raisonnable» (S. Besson, «La vulnérabilité et la structure des droits de l'homme... », p. 63). Aussi, "[p] our qu'un intérêt fondamental puisse être considéré comme suffisamment important pour donner naissance à un droit de l'homme [...] il faut pouvoir identifier un besoin général de protection de cet intérêt et, par conséquent, des menaces généralisées à cet intérêt» : c'est ce que l'auteure appelle la «menace égale ou vulnérabilité égale» (ibid., p. 64).

33. S. Besson, «La vulnérabilité et la structure des droits de l'homme...», p. 62.

34. Ibid.

35. L. Burgorgue-Larsen, «La vulnérabilité saisie par la philosophie, la sociologie et le droit...», p. 242.

36. A. Palanco, «Les variations autour des formes de vulnérabilité...», p. 33.

37. En psychologie, la résilience se définit comme l'«aptitude à affronter les épreuves, à trouver des ressources intérieures et des appuis extérieurs, à mettre en œuvre des mécanismes psychiques permettant de surmonter les traumatismes » (dictionnaire de l'Académie française, $9^{\mathrm{e}}$ éd.). Selon la Commission d'enrichissement de la langue française, qui consacre un site Internet aux termes publiés au Journal officiel de la République française, en sciences humaines et sociales, ce terme correspond davantage à la "capacité d'une personne ou d'une société à résister à une épreuve brutale et à en tirer parti pour se renforcer» (en ligne: http://www.culture.fr/franceterme).

38. R. Estupiñan-Silva, «La vulnérabilité saisie par la Cour interaméricaine», p. 92 et 110-113.

39. Sur la distinction entre «groupes» et «catégories» des personnes, voir ci-dessous.

40. M. Beloff, L. Clérico, «Derecho a condiciones de existencia digna y situación de vulnerabilidad en la jurisprudencia de la Corte Interamericana», Estudios constitucionales, vol. 14, $\mathrm{n}^{\circ}$ 1, 2016, p. 165-166. L'auteure précise ici que, sauf mention contraire, les traductions sont les siennes.

41. Sur cette différence, voir M. Rota, L'interprétation des Conventions américaine et européenne des droits de l'homme. Analyse comparée de la jurisprudence des deux Cours de protection des droits de l'homme, Issy-les-Moulineaux, LGDJ, 2018, p. 182-189. 
appelle la «fonction procédurale» de la vulnérabilité ${ }^{42}$. Le second procédé est l'octroi d'une protection spécifique à la personne vulnérable ${ }^{43}$. Pour ce faire, les Cours mettent à la charge des États des obligations positives qui vont audelà de celles habituellement imposées à l'égard de toute personne. S’agissant de la Cour européenne, il peut s'agir tant d'obligations de protection des personnes vulnérables, de mener une enquête lorsque leurs droits humains sont atteints mais aussi de prévention ${ }^{44}$, qui implique bien souvent une inversion de la charge de la preuve, reposant sur les États ${ }^{45}$. Elle peut aussi avoir vocation à limiter les droits et libertés d'autrui ${ }^{46}$, ou encore à abaisser le seuil de gravité qu'une atteinte à l'intégrité doit dépasser pour être qualifiée de torture ${ }^{47}$.

La Cour interaméricaine est quant à elle très claire: elle affirme dès 2006 dans son affaire Ximenes Lopes c. Brésil que

[...] toute personne en situation de vulnérabilité a droit à une protection spéciale, en raison des devoirs spéciaux dont l'accomplissement par l'État est nécessaire pour satisfaire aux obligations générales de respect et de garantie des droits de l'homme ${ }^{48}$.

Elle fait donc découler de l'article 1.1 de la Convention une obligation faite aux États « d'adopter des mesures positives, déterminables en fonction des besoins particuliers de protection du sujet de droit, que ce soit en raison de sa condition personnelle ou de la situation spécifique dans laquelle il se trouve ${ }^{49}$. Elle en fait découler des obligations positives foisonnantes, adaptées à chaque vulnérabilité et au contexte ${ }^{50}$ qui l'entoure et d'où il résulte une inversion systématique de la charge de la preuve au titre du devoir de prévention ${ }^{51}$.

Les Cours imposent donc aux États de remédier, d'apporter un palliatif à la situation de vulnérabilité dans laquelle se trouvent ces personnes, le but étant de leur permettre de jouir pleinement de leurs droits humains, de manière concrète et égale. Ceci aboutit à une prise en considération de l'individu situé, démarche commune aux deux Cours. Mais il ne faut cependant pas s'y tromper, car, s'il permet de «contrebalance[r] les effets néfastes de l'abstraction libérale de l'individu ${ }^{52}$, le recours à la vulnérabilité reste ancré dans la philosophie des droits attachée à chaque système.

\section{La vulnérabilité: un concept reflétant une conception différente des droits humains adoptée par les Cours}

Pour comprendre la philosophie des droits à laquelle se rattache chaque Cour, il faut rappeler que les Conventions qu'elles sont chargées de protéger ont toutes les deux été rédigées après la Deuxième Guerre mondiale, époque à laquelle les droits humains sont fondés non plus sur la liberté mais sur la dignité humaine. Ce concept peut néanmoins revêtir plusieurs significations ce qui explique une différence de conception des droits humains qui en découlent. Celle-ci rejaillit directement sur le concept de vulnérabilité qui fait l'objet d'une lecture qu'on peut qualifier de libérale s'agissant de la jurisprudence de la Cour européenne (A) et de sociale s'agissant de celle de la Cour interaméricaine (B).

\section{A. La lecture libérale de la vulnérabilité par la Cour européenne}

Si le concept de dignité se distingue de celui de vulnérabilité, un détour par son étude peut permettre de saisir les différences quant à l'appréhension de ce dernier. On peut à ce titre rappeler qu'une première lecture de la dignité consiste à l'attacher à toute personne humaine, qui, en raison de sa singularité, mérite respect ${ }^{53}$. C'est ici la qualité de personne humaine qui implique la reconnaissance de sa dignité et qui fonde la reconnaissance de droits. Le passage de la liberté à la dignité implique cependant un

42. S. Besson, «La vulnérabilité et la structure des droits de l’homme...», p. 77-78. Voir aussi B. Pastre-Belda, «La dimension responsabilisante de la vulnérabilité dans la jurisprudence de la Cour européenne des droits de l'homme", in La vulnérabilité en droit européen des droits de l'homme..., p. $165-171$.

43. Sur ce point, voir la récente et très complète étude de B. Pastre-Belda, «La dimension responsabilisante de la vulnérabilité....", p. 161-196.

44. Dans l'affaire Stubbings et autres c. Royaume-Uni, par exemple, la Cour européenne affirme que «les enfants et autres personnes vulnérables, en particulier, ont droit à la protection de l'État, sous la forme d'une prévention efficace» (Cour EDH, 22 octobre 1996, Stubbings et autres c. Royaume-Uni, $\mathrm{n}^{\circ} 22083 / 93$ et $22095 / 93, \$ 64$ ).

45. S. Besson, «La vulnérabilité et la structure des droits de l'homme... », p. 78.

46. Comme le constate fort justement Jean-Yves Carlier à propos de l'affaire Dudgeon c. Royaume-Uni, «ce premier arrêt, en faisant référence aux "personnes spécialement vulnérables", ne se fonde nullement sur cette vulnérabilité pour condamner une atteinte aux droits fondamentaux du requérant, homosexuel, mais, à l'inverse, pour admettre qu'une limite, proportionnée, à ces droits fondamentaux serait possible» (J.-Y. Carlier, "Des droits de l'homme vulnérable...", p. 184)

47. S. Besson, «La vulnérabilité et la structure des droits de l'homme... », p. 76.

48. Cour IDH, 4 juillet 2006, Ximenes Lopes c. Brésil, fond, réparations et frais, série C, $\mathrm{n}^{\circ} 149, \$ 103$.

49. Ibid. Voir à cet égard B. Duhaime, «Le système interaméricain et la protection des droits économiques, sociaux et culturels des personnes et des groupes vivant dans des conditions particulières de vulnérabilité», Annuaire canadien de droit international, n 44, 2006, p. 95-160.

50. Sur l'importance du contexte, se référer à nos propos conclusifs, infra.

51. Pour un exemple récent et a contrario (qui fait figure d'exception dans la jurisprudence de la Cour), voir Cour IDH, 21 novembre 2019 , Virula et autres c. Guatemala, exception préliminaire, fond, réparations et frais, série C, n $393, \S 55-60$.

52. S. Besson, «La vulnérabilité et la structure des droits de l’homme...», p. 6o.

53. On peut ici relier cette conception au sens que lui attribue Kant, selon lequel la dignité est attachée à la personne en raison de son caractère irremplaçable (E. Kant, Fondements de la métaphysique des mœeurs, V. Delbos (trad.), Paris, Delagrave, 1969, p. 160). 
changement de conception quant au titulaire des droits car son objectif principal est de reconnaître l'aspect social de l'être humain. La dignité est alors attachée à la personne, envisagée au sein d'une société, de la communauté humaine, et non pas à l'individu, «être générique et non situé » ${ }^{54}$, envisagé seul contre l'État. Aussi, le titulaire des droits n'est plus l'individu ou encore l'être humain du siècle des Lumières, mais la personne, concept englobant sa réalité sociale 55 .

Cependant, ce passage de la liberté à la dignité en tant que fondement des droits n'implique pas nécessairement une remise en cause du libéralisme et de l'individualisme. Comme le constate Xavier Bioy, «le concept de personne peut [...] se percevoir comme son prolongement, certes comme correcteur de dérives, mais non comme étranger à l'individualisme ${ }^{56}$. En effet, c'est bien le « concept unitaire de personne ${ }^{57}$ qui est pris en considération, la dignité y étant avant tout attachée, et pas à l'humanité, groupe auquel elle appartient. Aussi, le titulaire des droits reste le «sujet libéral», mais qui diffère de celui pris en compte par la philosophie des Lumières en raison de son caractère «situé ${ }^{58}$.

Ainsi comprise, la dignité suppose de respecter la liberté de l'homme et établit même un lien entre ces deux concepts $^{59}$. Le nécessaire respect de la dignité de l'homme n'écarte pas la liberté de ce dernier et c'est en cela qu'on peut affirmer que «la dignité exige la liberté mais la liberté n'est pas toute la dignité ${ }^{60}$. Elle ne s'inscrit plus directement dans la philosophie libérale, mais s'en accommode.

C'est cette conception qu'adopte clairement la Cour européenne en soulignant, dans l'affaire S. W.c. RoyaumeUni, que «l'essence même [de la Convention] est le respect de la dignité et de la liberté humaines ${ }^{61}$. Car, si la dignité est reconnue, la Cour prend à chaque fois le soin de préciser que la liberté l'est aussi, et au même titre. En mettant sur un pied d'égalité les deux concepts, elle explique aussi les droits par la volonté de préserver le pouvoir d'autodétermination de l'homme, qui doit pouvoir choisir son propre comportement. Elle semble éviter l'écueil de l'interprétation antimoderne de la dignité, tout en se laissant la possibilité de reconnaître une portée plus étendue des droits, nécessitée par le respect de la dignité humaine. Cette référence à la dignité implique cependant une prise en considération de la personne au sein de la société et par conséquent de sa potentielle vulnérabilité. Il faut en effet avoir à l'esprit que la vulnérabilité est avant tout un concept relationnel: « un individu est susceptible d'une atteinte, alors qu'un autre en porte la menace et l'un implique l'autre ${ }^{62}$.

L'attachement de la Cour européenne au libéralisme explique qu'elle préfère raisonner en termes de catégories qu'en termes de groupes de personnes. Et pour cause: l'«approche catégorielle a pour caractéristique générale de mettre en évidence des spécificités qui doivent être prises en compte dans l'application de la norme, sans pour autant briser fondamentalement l'unité du sujet des droits de l'homme ${ }^{63}$. Contrairement aux catégories de personnes qui «ont quelque chose de conjoncturel, de volatil, d'instable et d'abstrait, les groupes d'appartenance ont à l'inverse une essence, une substance concrète, une permanence ${ }^{64}$. Comme le souligne aussi Yaël Attal-Galy, «la catégorie rassemble des individus isolés, alors que les membres d'un groupe, défini par leur appartenance religieuse ou ethnique, revendiquent des droits propres à l'exercice et à l'existence de leur groupe ${ }^{65}$. C'est pourquoi, par exemple, la Cour refuse de qualifier les femmes de groupe vulnérable ${ }^{66}$.

On observe cependant un certain glissement de la jurisprudence européenne qui passe par la reconnaissance de discriminations indirectes ${ }^{67}$ à l'égard de groupes qualifiés de vulnérables, tels que les Roms / Tsiganes. La Cour souligne en effet que leur vulnérabilité implique «d'accorder une attention spéciale à leurs besoins et à leur mode de vie propre tant dans le cadre réglementaire considéré que lors de la prise de décision dans des cas

54. J. Morange, Manuel de droits de l'homme et libertés publiques, Paris, Presses universitaires de France, 2007, p. 36.

55. X. Bioy, Le concept de personne humaine en droit public. Recherche sur le sujet des droits fondamentaux, Paris, Dalloz, $2003, \mathrm{p} .737$.

56. Ibid., p. 748

57. Ibid., p. 747

58. Nous reprenons ici les termes de Xavier Bioy, à qui revient la paternité de l'expression «sujet libéral situé» (X. Bioy, Le concept de personne humaine en droit public..., p. 747).

59. Voir sur ce point O. Cayla, «Le coup d'État de droit?», Le débat, n 100,1998 , p. 125 ou encore C. Girard, S. Hennette-Vauchez, «Introduction», in La dignité de la personne humaine: recherche sur un processus de juridicisation, C. Girard, S. Hennette-Vauchez (dir.), Paris, Presses universitaires de France, 2005, p. 26-33.

60. B. Maurer, Le principe de respect de la dignité humaine et la Convention européenne des droits de l'homme, Paris, La documentation française, 1999, p. 48.

61. Cour EDH, 22 novembre 1995, S. W. c. Royaume-Uni, n² 20166/92, \$ 44 repris par Cour EDH, 11 juillet 2002, Goodwin c. Royaume-Uni, $\mathrm{n}^{\circ} 28957 / 95, \S 90$.

62. S. Besson, «La vulnérabilité et la structure des droits de l'homme...», p. 60

63. C. Ruet, «La vulnérabilité...».

64. D. Lochak, "Les minorités et le droit public français: du refus des différences à la gestion des différences", in Les minorités et leurs droits depuis 1789, A. Fenet, G. Soulier (dir.), Paris, L'Harmattan, 1989, p. 113

65. Y. Attal-Galy, Droits de l'homme et catégories d'individus, Paris, LGDJ, 2003, p. 286

66. A. Palanco, «Les variations autour des formes de vulnérabilité...», p. 48. Voir M. Rota, L’interprétation des Conventions..., p. 396.

67. Peut-être faut-il ici préciser qu'on parle de discrimination indirecte lorsque ce n'est pas l'État qui va marginaliser ou mettre à l'écart certaines personnes sans justifications raisonnables, objectives et proportionnées (définition de la discrimination directe), mais que c'est un état de fait : l'existence de situations d'inégalités de fait implique cette marginalisation injustifiée (discrimination indirecte, liée à la rupture de l'égalité substantielle). Sur la reconnaissance des discriminations indirectes par la Cour européenne des droits de l'homme, voir M. Rota, L'interprétation des Conventions..., p. 394-395. 
particuliers ${ }^{68}$ et donc une intervention active de la part de l'État pour y mettre fin. Mais ce glissement s'arrête à ce stade. Contrairement à la Cour interaméricaine, la Cour européenne refuse de consacrer des discriminations positives à l'égard de groupes vulnérables ${ }^{69}$. Elle refuse aussi de reconnaître des droits humains à ces groupes vulnérables qui, en tant que tels, mériteraient respect ${ }^{70}$. Une telle reconnaissance risquerait de limiter la liberté de l'individu au nom du nécessaire respect dû non plus à la personne humaine mais audit groupe. Il en va complètement différemment de la jurisprudence de la Cour interaméricaine.

\section{B. La lecture sociale de la vulnérabilité par la Cour interaméricaine}

La Cour interaméricaine adopte une vision plus sociale des droits humains. Cela s'explique, là encore, par le concept philosophique qu'elle place à leur fondement: la dignité humaine, mais qui n'est plus attachée à la personne, mais à l'être humain, entendu dans son sens générique. La dignité lui est reconnue en raison de son appartenance à l'humanité, qui devient son véritable dépositaire. Dans la mesure où elle est attachée à la personne humaine - entendue comme telle en tant que membre de ce groupe et que c'est ce groupe qui mérite respect - elle devient «une qualité opposable à l'homme par des tiers $»^{71}$. Elle peut de ce fait être opposée à la liberté, "au nom de la dignité en soi», c'est-à-dire de «la protection de l'humanité que renferme la personne du requérant $»^{72}$.

Comprise ainsi, la dignité peut se rattacher à une vision dite «communautaire» des droits humains. Le fait d'attacher la dignité à un groupe implique en effet que les droits n'appartiennent plus à l'individu mais à une collectivité et qu'ils soient exercés dans son intérêt ${ }^{73}$. Cette vision collective de la dignité et des droits qui en découlent explique qu'on puisse reconnaître d'autres groupes, venant tout autant limiter la liberté d'un sujet y appartenant, au nom d'un intérêt collectif. On peut alors voir dans cette conception une résurgence de la dignitas de la Grèce et de la Rome antique, où la dignité était attachée à une institution, à un rang ou à une fonction officielle ou corporative. Le groupe ou la communauté en question se voyant reconnaître un statut particulier pourra requérir de ses membres d'agir de manière compatible avec sa "dignité». La liberté de toute personne peut alors être réduite et «le juge [devient] dans tous les cas [...] celui qui exerce en dernière instance cette fonction de représentation des valeurs communautaires ${ }^{74}$.

La Cour interaméricaine consacre directement cette vision. Dès sa première décision portant sur le fond elle affirme en effet que «l'exercice de la fonction publique a des limites qui découlent du fait que les droits de l'homme soient des attributs inhérents à la dignité humaine et, par conséquent, supérieurs au pouvoir de l'État ${ }^{75}$. La dignité est ici opposée au pouvoir de l'État, comme l'était la liberté dans les déclarations occidentales. Elle fonde les droits humains - ils sont des attributs qui lui sont inhérents - et limite la puissance étatique. Il s'agit là d'un présupposé puisque, selon la Cour, «le fait que toute personne ait des attributs inviolables et inhérents à sa dignité humaine est indiscutable $»^{76}$. La liberté n'est, quant à elle, pas entendue en tant que concept au fondement des droits: il s'agit d'un simple «droit de l'homme " 77 , auquel la Cour reconnaît en revanche une nature particulière ${ }^{78}$. Il faut en effet rappeler que la dignité ne s'oppose pas forcément à la liberté; mais elle la fonde et peut l'encadrer.

Il en va différemment pour le principe d'égalité. Dans son quatrième avis consultatif, la Cour souligne que «la notion d'égalité découle directement de l'unité du genre humain et est inséparable de la dignité essentielle de la personne, avec laquelle toute situation [discriminatoire] est incompatible ${ }^{79}$. Or, dans la mesure où elle est «inséparable» de la dignité, l'égalité en est l'expression même. La différence entre la dignité de la personne humaine et l'égalité est alors très ténue. En consacrant la dignité comme fondement, les juges partent du postulat initial

68. Cour EDH, GC, 13 novembre 2007, D. H. et autres c. République tchèque, nº 57325/00, $\$ 181$.

69. La Cour refuse en effet d'imposer aux États d'agir pour réduire les inégalités de fait en octroyant aux personnes de facto discriminées «des droits ou des avantages dérogatoires et préférentiels" (définition de la discrimination positive de Y. Attal-Galy, Droits de l'homme..., p. 280; reprise dans M. Rota, L'interprétation des Conventions..., p. 396).

70. M. Rota, L'interprétation des Conventions..., p. 396-397.

71. S. Hennette-Vauchez, «La dignité en $3 \mathrm{D}$ : analyses», in Voyage au bout de la dignité: recherche généalogique sur le principe juridique de dignité de la personne humaine, C. Girard, S. Hennette-Vauchez (dir.), Paris, Mission de recherche droit et justice, 2004, p. 30.

72. Ibid., p. 31 .

73. Se reporter à C. Girard, S. Hennette-Vauchez, «Introduction», p. 30.

74. O. Cayla, «Le coup d'État de droit?», p. 128.

75. Cour IDH, 29 juillet 1988, Velásquez Rodríguez c. Honduras, fond, série C, $\mathrm{n}^{\circ} 4, \$ 165$.

76. Cour IDH, 17 septembre 2003, Condición Jurídica y Derechos de los Migrantes Indocumentados, opinion consultative $\mathrm{n}^{\circ}$ OC-18/o3, série A, $\mathrm{n}^{\circ}$ 18, $\$ 73$. Voir à ce sujet H. Nogueira Alcalá, «Los derechos esenciales o humanos contenidos en los Tratados Internacionales y su ubicación en el ordenamiento jurídico nacional: doctrina y jurisprudencia», Ius et Praxis, vol. 9, nº 1, 2003, p. 403-466. Voir également W. G. Di Lorenzo, «Abertura da constituição", Direito e Justiça, vol. 24, nº 2, 2001, p. 171-200; H. Alves da Frota, "O princípio da dignidade da pessoa humana à luz do direito constitucional comparado e do direito internacional dos direitos humanos », Revista Latinoamericana de Derecho, année 2, $\mathrm{n}^{\circ}$ 4, $2005, \mathrm{p} .1-26$.

77. Cour IDH, 21 novembre 2007, Chaparro Álvarez et Lapo Íñiguez. c. Équateur, exceptions préliminaires, fond, réparations et frais, série C, nº 170 , $\$ 52$.

78. C'est en effet, selon elle, «un droit de l'homme de base, caractéristique des attributs de la personne, qui se projette dans toute la Convention» (ibid.).

79. Cour IDH, 19 janvier 1984, Propuesta de Modificación a la Constitución Política de Costa Rica Relacionada con la Naturalización, opinion consultative $\mathrm{n}^{\circ} \mathrm{OC}-4 / 84$, série $\mathrm{A}, \mathrm{n}^{\circ} 4, \$ 55$. Elle en déduit dès lors que les principes «d'égalité et de non-discrimination découlent de l'idée de l'unité de la dignité et de la nature de la personne [humaine]» (ibid., §56). 
que tout homme parce qu'il est homme mérite respect. C'est pour respecter cette essence humaine que les droits sont reconnus. L'égalité est quant à elle supposée dans cette appartenance au genre humain (c'est parce que toute personne appartient au genre humain qu'elle est égale à toute autre), et en est donc l'expression la plus fondamentale ${ }^{80}$. Les autres droits, dont la liberté, en découlent ensuite. On comprend alors la position de la Cour qui reconnaît la valeur de jus cogens attachée à ce principe ${ }^{81}$. C'est parce que le principe d'égalité est l'expression même de la dignité humaine, qui elle-même limite le pouvoir de l'État, que le principe d'égalité devient la base de tout ordonnancement juridique.

Or, cette dignité égalité va expliquer une reconnaissance de droit et libertés spécifiquement attribués à des groupes vulnérables via la reconnaissance des discriminations indirectes par exemple, ce que fait la Cour interaméricaine depuis beaucoup plus longtemps et de manière beaucoup plus systématique que la Cour européenne $^{82}$. Elle passe aussi et surtout par la reconnaissance de discriminations positives dont doivent bénéficier les groupes se trouvant justement en situation défavorable vis-à-vis des autres groupes composant la société et, donc, en position de vulnérabilité ${ }^{83}$. Enfin, la Cour n'hésite pas à reconnaître des droits à des groupes vulnérables, méritant un égal respect ${ }^{84}$. À ce titre, la vulnérabilité devient une condition objective d'une personne, en tant que membre d'un groupe vulnérable, qui est lui-même le produit d'une relation déséquilibrée vis-à-vis des autres groupes qui composent la société.
Comprise comme telle, l'utilisation de la vulnérabilité peut facilement faire réapparaître les «risques» - différenciation, essentialisation et paternalisme - identifiés par la doctrine occidentale, attachée à la liberté de l'individu. Il nous semble cependant qu'à partir du moment où l'autonomie personnelle - qui est d'ailleurs au cœur du concept de résilience - est reconnue et valorisée par la Cour, ces risques peuvent être surmontés. Or la Cour se réfère justement la première fois à l'autonomie personnelle dans la décision Ximenes Lopes c. Brésil précitée ${ }^{85}$ qui consacre son dictum relatif à la vulnérabilité ${ }^{86}$. Aussi peut-on y voir là la tentative de mise en place d'une garantie contre ces dérives potentielles. Elle passerait par la recherche d'un juste équilibre entre garantie de l'égalité substantielle ( $v i a$ la reconnaissance de la vulnérabilité) et de la liberté individuelle (via la reconnaissance de l'autonomie personnelle).

Dans la mesure où, cependant, il revient au juge de placer le curseur sur l'une ou l'autre des deux valeurs, elle est nécessairement soumise à critique. C'est pourquoi l' «angle d'analyse» proposé par Laurence BurgorgueLarsen "consistant à plus et mieux valoriser les contextes de vulnérabilité, plutôt que les personnes et / ou les groupes vulnérables ${ }^{87}$ nous paraît particulièrement pertinent. Il nécessite cependant une ouverture du droit à ce dont il est le produit, i. e. la société, et à une remise en cause du positivisme juridique, là encore bien ancré dans la pensée occidentale. C'est peut-être aussi pourquoi la Cour européenne fait si peu souvent référence au contexte de chaque affaire, contrairement à ce que fait la Cour interaméricaine $^{88}$, qui pourrait ici servir de modèle.

80. En effet, la dignité «privilégie le présupposé de l'égalité entre les personnes humaines et permet une égale reconnaissance» (C. Girard, S. HennetteVauchez, «La dignité en $3 \mathrm{D}$ », p. 28).

81. Cour IDH, 17 septembre 2003, Condición Jurídica y Derechos de los Migrantes Indocumentados, $\$ 101$.

82. M. Rota, L'interprétation des Conventions..., p. 394-395.

83. Ibid., p. 395-396.

84. Ibid., p. 396-40o.

85. Cour IDH, 4 juillet 2006, Ximenes Lopes c. Brésil, $\$ 130$.

86. Voir supra.

87. L. Burgorgue-Larsen, «La vulnérabilité saisie par la philosophie, la sociologie et le droit... », p. 242.

88. Comme le rappelle Laurence Burgorgue-Larsen: «la jurisprudence interaméricaine [...] a toujours attaché une place centrale aux contextes (historiques, sociologiques, culturels, etc.)» (ibid., p. 242). L’importance du contexte dans la jurisprudence de la Cour interaméricaine est également régulièrement soulignée par les juges de cette même Cour. Voir, pour un exemple récent, l’opinion du président de la Cour Eduardo Ferrer Mac-Gregor Poisot et de la juge Elizabeth Odio Benito sous l'affaire Cour IDH, 19 novembre 2019, Díaz Loreto et autres c. Venezuela, exceptions préliminaires, fond, réparations et frais, série $C, n^{\circ} 392$. 